\title{
Uniquely monopolar-partitionable block graphs
}

\author{
Xuegang Chen ${ }^{1,2 \mid *}$ and Jing Huang $\|^{*}$ \\ ${ }^{1}$ Department of Mathematics, North China Electric Power University, Beijing 102206, China \\ ${ }^{2}$ Department of Applied Mathematics, Beijing University of Technology, Beijing 100124, China \\ ${ }^{3}$ Department of Mathematics and Statistics, University of Victoria, B.C., Canada
}

received $27^{\text {th }}$ Dec. 2013, revised $18^{\text {th }}$ Apr. 2014, accepted $20^{\text {th }}$ Apr. 2014.

As a common generalization of bipartite and split graphs, monopolar graphs are defined in terms of the existence of certain vertex partitions. It has been shown that to determine whether a graph has such a partition is an NPcomplete problem for general graphs, and is polynomial time solvable for several classes of graphs. In this paper, we investigate graphs that admit a unique such partition and call them uniquely monopolar-partitionable graphs. By employing a tree trimming technique, we obtain a characterization of uniquely monopolar-partitionable block graphs. Our characterization implies a polynomial time algorithm for determining whether a block graph is uniquely monopolar-partitionable.

Keywords: Monopolar graph, monopolar partition, uniquely monopolar-partitionable grap, block graph, characterization, polynomial time algorithm

\section{Introduction}

Given a graph $G$, a monopolar partition of $G$ is a partition $(A, B)$ of its vertex set where $A$ is an independent set and $B$ induces a disjoint union of cliques in $G$. A graph which admits a monopolar partition is called monopolar or monopolar-partitionable.

Monopolar graphs were introduced in [17] as a common generalization of bipartite graphs and split graphs. Every bipartition of a bipartite graph is a monopolar partition. Graphs which admit monopolar partitions $(A, B)$ where $B$ induces a single clique are precisely split graphs [12, 14].

A monopolar graph is called uniquely monopolar-partitionable if it has exactly one monopolar partition, that is, if $(A, B)$ and $\left(A^{\prime}, B^{\prime}\right)$ are both monopolar partitions of $G$ then $A=A^{\prime}$ (and $B=B^{\prime}$ ). Since each complete graph has two monopolar partitions $(A, B)$ and $\left(A^{\prime}, B^{\prime}\right)$ where $A$ and $A^{\prime}$ are the empty set and singleton set respectively, no complete graph is uniquely monopolar-partitionable. On the other hand, the graph obtained from two complete graphs of order at least three by identifying two vertices, one from each, is uniquely monopolar-partitionable.

\footnotetext{
*Email: gxcxdme163.com. Research supported by Beijing Postdoctoral Research Foundation.

†Email: huangj@uvic. ca. Research supported by NSERC.
} 
Unlike bipartite graphs and split graphs which are easy to recognize, recognizing monopolar graphs in general is an NP-complete problem (cf. [2] and [11]). It is currently unknown whether uniquely monopolar-partitionable graphs are recognizable in polynomial time.

In this paper, we consider the uniqueness of monopolar partitionability of block graphs. A block of a graph $G$ is a maximal subgraph of $G$ without cut-vertices. A graph is a block graph if every block is a clique (cf. [1, 15]). We shall give a structural characterzation of uniquely monopolar-partitionable block graphs by using a tree trimming technique. As a by-product, we obtain a polynomial time algorithm for determining whether a block graph is uniquely monopolar-partitionable. We note that such an algorithm can be extracted from [8].

\section{Basic definitions}

We follow the standard definition and terminology from [18] and consider only simple graphs. Let $G=$ $(V, E)$ be a graph. The neighbourhood $N(v)$ of a vertex $v$ in $G$ consists of all vertices adjacent to $v$. The size of $N(v)$ is the degree of $v$ and denoted by $d(v)$. If $d(v)=1$, then vertex $v$ is called a leaf of $G$. The closed neighhood $N[v]$ of $v$ is $N(v) \cup\{v\}$. For any $S \subseteq V$, the subgraph of $G$ induced by $S$ is denoted by $G[S]$. For convenience we write $G-S=G[V-S]$. For any two vertices $u, v \in V$, the distance $d_{G}(u, v)$ between $u$ and $v$ in $G$ is the length of a shortest $u, v$-path in $G$, and the diameter $\operatorname{diam}(G)$ of $G$ is the maximum distance of any two vertices in $G$. We shall use $P_{n}, C_{n}$ and $K_{n}$ to denote the path, cycle and clique with order $n$, respectively.

Let $H$ be a subgraph of a graph $G$. By contracting $H$ in $G$ we mean to obtain a new graph $G^{\prime}$ from $G-V(H)$ by adding a new vertex $w$ adjacent to all vertices which have at least one neighbour in $H$.

Let $G_{1}, G_{2}, \cdots, G_{t}$ be the components of $G-v$. For any $1 \leq i_{1}<i_{2}<\cdots<i_{s} \leq t, R_{v}^{s}=$ $G\left[V\left(\bigcup_{1 \leq j \leq s} G_{i_{j}}\right) \cup\{v\}\right]$ is called a tangent subgraph of $G$. If a star with order at least two is a component of $G-v$ and only one center of the star is adjacent to $v$, then the star is called an end star of $G$, and $v$ is said to be adjacent to the end star. The vertex of the star that is adjacent to $v$ is taken as the center of the end star.

Let $G$ be a block graph with at least two blocks and let $Q$ be a block of $G$. A vertex $v$ of $G$ is said to be adjacent to $Q$ if $v$ is not in $Q$ but adjacent to a vertex of $Q$. If the order of $Q$ is at least 3, then $Q$ is called a big block of $G$. If a big block $Q$ contains a unique cut vertex and $G-Q$ is connected, then $Q$ is called a terminal block; if the big block $Q$ contains a unique cut vertex and $G-Q$ is disconnected, then $Q$ is called a suspending block of $G$. If two big blocks have no common vertex but contain adjacent vertices, then the two blocks are called adjacent. If two big blocks $Q_{1}$ and $Q_{2}$ have a common vertex $z$. then we call the subgraph $G\left[V\left(Q_{1} \cup Q_{2}\right)\right]$ a bowtie of $G$ and the common vertex $z$ is the center of the bowtie. The bowtie is called a terminal bowtie if $G-Q_{1}-Q_{2}$ is connected and its center is adjacent to exactly one vertex $w$ of $V(G)-V\left(Q_{1} \cup Q_{2}\right)$. Vertex $w$ is said to be adjacent to the terminal bowtie. If the center of an end star or a terminal bowtie is adjacent to a big block, then the end star or the terminal bowtie is said to be adjacent to the big block. Two terminal bowties are adjacent if their centers are adjacent. A big block is said to be adhered to a vertex $v$ if $v$ is identified with a vertex from the block. Adhering a bowtie to a vertex $v$ means adhering two big blocks to the vertex. If a block graph $G$ is induced by $t \geq 3$ big blocks with a common vertex, then $G$ is called a flower.

Let $G^{\prime}$ be an induced subgraph of $G$. Suppose that $\left(A^{\prime}, B^{\prime}\right)$ is a monopolar partition of $G^{\prime}$. If there is a monopolar partition $(A, B)$ of $G$ such that $A \supseteq A^{\prime}$ and $B \supseteq B^{\prime}$, then we say that the monopolar partition $\left(A^{\prime}, B^{\prime}\right)$ can be extended to a monopolar partition of $G$. If there is a unique monopolar partition $(A, B)$ 
of $G$ such that $A \supseteq A^{\prime}$ and $B \supseteq B^{\prime}$, then we say that the monopolar partition $\left(A^{\prime}, B^{\prime}\right)$ can be extended to exactly one monopolar partition of $G$.

\section{Basic properties}

Suppose that $G$ is a uniquely monopolar-partitionable graph and $(A, B)$ is the unique monopolar partition of $G$. For any vertex $v \in V(G)$, if $G[N(v)]$ has an induced $P_{3}$, then $v \in B$. If $G[N(v)]$ has no induced $P_{3}$, then $G[N(v)]$ is a disjoint union of cliques. If $G[N(v)]$ contains two cliques $Q_{1}$ and $Q_{2}$ such that each has at least two vertices, then $v \in A$.

Proposition 3.1 Let $T$ be a tree with order $n \geq 2$. For any edge $u v \in E(T)$, there exists a monopolar partition $(A, B)$ such that $u, v \in B$.

Proof: Let $T^{\prime}$ be obtained from $T$ by contracting edge $u v$ and let $w$ denote the new vertex of $T^{\prime}$. Then $T^{\prime}$ is a tree. Let $\left(A^{\prime}, B^{\prime}\right)$ be the bipartition of $T^{\prime}$. Say $w \in B^{\prime}$. Let $(A, B)=\left(A^{\prime},\left(B^{\prime} \backslash\{w\}\right) \cup\{u, v\}\right)$. Then $(A, B)$ is a monopolar partition of $T$ such that $u, v \in B$.

Corollary 3.2 No tree is uniquely monopolar-partitionable.

Proposition 3.3 Let $G$ be a uniquely monopolar-partitionable graph and $v$ be a vertex of $G$. Suppose that $C$ is a component of $G-v$ and $V(C) \cup\{v\}$ induces a tree in $G$. Then $V(C) \cup\{v\}$ induces a star in $G$.

Proof: Since $V(C) \cup\{v\}$ induces a tree, $v$ has a unique neighbour in $C$ which we denoted by $x$. We show that $x$ is adjacent to every other vertex in $C$. Suppose not. Then there exist vertices $y, z$ such that $x y z$ is a path in $C$. Since $G$ is a monopolar graph and $G-C$ is a subgraph of $G, G-C$ is a monopolar graph. Let $\left(A^{\prime}, B^{\prime}\right)$ be a monopolar partition of $G-C$.

Assume first that $v \in A^{\prime}$. By Proposition 3.1. $C$ has a monopolar partition $\left(A_{1}, B_{1}\right)$ such that $x, y \in$ $B_{1}$. Let $\left(A_{2}, B_{2}\right)$ be a bipartition of $C$ where $x \in B_{2}$ and $y \in A_{2}$. Then $\left(A^{\prime} \cup A_{1}, B^{\prime} \cup B_{1}\right)$ and $\left(A^{\prime} \cup A_{2}, B^{\prime} \cup B_{2}\right)$ are different monopolar partitions of $G$, which is a contradiction.

Assume now that $v \in B^{\prime}$. By Proposition 3.1. $C$ has a monopolar partition $\left(A_{1}, B_{1}\right)$ such that $y, z \in$ $B_{1}$. Let $\left(A_{2}, B_{2}\right)$ be a bipartition of $C$ where $x, z \in A_{2}$ and $y \in B_{2}$. Then $\left(A^{\prime} \cup A_{1}, B^{\prime} \cup B_{1}\right)$ and $\left(A^{\prime} \cup A_{2}, B^{\prime} \cup B_{2}\right)$ are different monopolar partitions of $G$, which is a contradiction.

Suppose that $C$ is a component of $G-v$ of order at least two and $G[V(C) \cup\{v\}]$ is a tree. By Proposition 3.3, if $G$ is a uniquely monopolar-partitionable graph, then $C$ is an end star of $G$.

For any monopolar partition $(A, B)$ of $G$, the center of a bowtie must belong to $A$. Hence, we have the following.

Proposition 3.4 Let $Q_{i}$ be a big block of block graph $G$ for $i=1,2,3$. If $G\left[V\left(Q_{1} \cup Q_{2}\right)\right]$ and $G\left[V\left(Q_{2} \cup Q_{3}\right)\right]$ are two bowties of $G$ with different centers, then $G$ has no monopolar partition.

Proposition 3.5 Let $Q_{1}, \cdots, Q_{t}$ be big blocks of block graph $G$ containing vertex $u$ and $t \geq 2$. Let $\widehat{G}=G-V\left(\bigcup_{1 \leq j \leq t} Q_{j}\right), S_{1}=N(u) \cap V(\widehat{G})$, and $S_{2}=N\left(V\left(\bigcup_{1 \leq j \leq t} Q_{j}\right)-u\right) \cap V(\widehat{G})$. Assume $S_{1} \cup S_{2} \neq \emptyset$. Let $G^{\prime}$ be obtained from $\widehat{G}$ by the following two operations:

- For every $w \in S_{1}$, adding a bowtie and joining its center to $w$; 
- For every $w \in S_{2}$, adhering a bowtie to $w$.

Then $G$ is uniquely monopolar-partitionable if and only if $G^{\prime}$ is.

Proof: Suppose that $G$ is uniquely monopolar-partitionable. Since $\widehat{G}$ is a subgraph of $G, \widehat{G}$ is a monopolar graph. The monopolar partition of $G$, when restricted to $\widehat{G}$, can be extended to a monopolar partition of $G^{\prime}$. Hence $G^{\prime}$ is a monopolar graph. Assume that $G^{\prime}$ has at least two different monopolar partitions. For any monopolar partition $\left(A^{\prime}, B^{\prime}\right)$ of $G^{\prime}$, it is obvious that $S_{1} \subseteq B^{\prime}$ and $S_{2} \subseteq A^{\prime}$. So, $\widehat{G}$ has at least two different monopolar partitions. Furthermore, each monopolar partition of $\widehat{G}$ can be extended to a monopolar partition of $G$. Hence $G$ has at least two different monopolar partitions, which is a contradiction. Hence $G^{\prime}$ is uniquely monopolar-partitionable.

Suppose that $G^{\prime}$ is uniquely monopolar-partitionable. It is obvious that $G$ is a monopolar graph. For any monopolar partition $(A, B)$ of $G, S_{1} \subseteq B$ and $S_{2} \subseteq A$. If $G$ has at least two different monopolar partitions, then $\widehat{G}$ has at least two different monopolar partitions. Since each monopolar partition of $G$, when restricted to $\widehat{G}$, can be extended to a monopolar partition of $G^{\prime}, G^{\prime}$ has at least two different monopolar partitions, which is a contradiction. Hence $G$ is uniquely monopolar-partitionable.

By Proposition 3.5. we can assume that block graph $G$ has no three big blocks with a common vertex. Moreover, each bowtie of $G$ is a terminal bowtie. A proof similar to that of Proposition 3.5 yields the following.

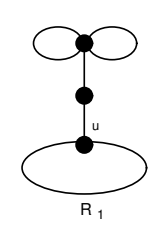

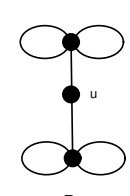

$\mathrm{R}_{2}$

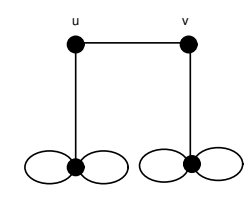

$\mathrm{R}_{3}$

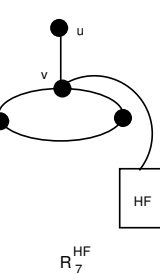

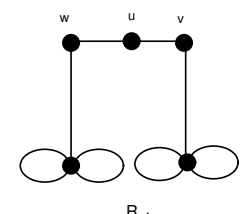

$\mathrm{R}_{4}$

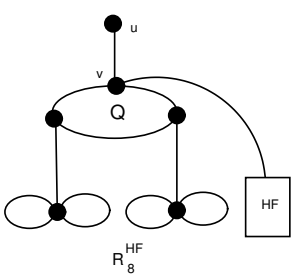

Fig. 1. Each ellipse is a big block, each vertex of $Q \backslash\{v\}$ in $R_{5} \cup R_{6}$ is adjacent to exactly one terminal bowtie, and $H F$ is a subgraph of $G$.

Proposition 3.6 Let $G$ be a block graph and let $G^{\prime}$ be defined as follows:

- Suppose that $G$ contains induced subgraph $R_{1}$. Let $G^{\prime}$ be obtained from $G$ by deleting the terminal bowtie of $R_{1}$ and adhering a big block to vertex $u$;

- Suppose that $G$ contains induced subgraph $R_{2}$. Let $G^{\prime}$ be obtained from $G$ by deleting a terminal bowtie of $R_{2}$; 
- Suppose that $G$ contains induced subgraph $R_{3}$, where $V(G)-V\left(R_{3}\right) \neq \emptyset$ and $N(u) \cap N(v)=\emptyset$. Let $G^{\prime}$ be obtained from $G$ by deleting $R_{3}$ and adhering a bowtie to each vertex $w \in(N(u) \cup$ $N(v)) \backslash V\left(R_{3}\right)$

- Suppose that $G$ contains induced subgraph $R_{4}$. Let $G^{\prime}$ be obtained from $G$ by deleting the two terminal bowties of $R_{4}$ and adhering a bowtie to vertex $u$;

- Suppose that $G$ contains induced subgraph $R_{5}$ and $V(G)-V\left(R_{5}\right) \neq \emptyset$. Let $G^{\prime}$ be obtained from $G$ by deleting $R_{5}$ and adhering a bowtie to each vertex of $N(Q) \backslash V\left(R_{5}\right)$.

Then $G$ is uniquely monopolar-partitionable if and only if $G^{\prime}$ is.

Proposition 3.7 Suppose that $(A, B)$ is the unique monopolar partition of block graph $G$.

(1) If $Q$ is either a terminal block or a suspending block, then the cut vertex $v$ of $Q$ belongs to A;

(2) The center $x$ of each end star belongs to $A$.

Proof: (1) Suppose that $v \in B$. If $A \cap V(Q)=\emptyset$, say $u \in V(Q) \backslash\{v\}$, then $(A \cup\{u\}, B \backslash\{u\})$ is a monopolar partition of $G$. If $A \cap V(Q) \neq \emptyset$, say $u \in A \cap V(Q)$, then $(A \backslash\{u\}, B \cup\{u\})$ is a monopolar partition of $G$. Hence, if $v \in B$, then $G$ has a monopolar partition different from $(A, B)$, which is a contradiction. So, $v \in A$.

(2) Assume that $v x \in E(G)$ and $v$ does not belong to the end star. Suppose that $x \in B$. If $v \in B$, then $((A-N(x)) \cup\{x\},(B \backslash\{x\}) \cup N(x))$ is a monopolar partition of $G$. Suppose that $v \in A$. If $B \cap N(x)=\emptyset$, say $w \in N(x) \backslash\{u\}$, then $(A \backslash\{w\}, B \cup\{w\})$ is a monopolar partition of $G$. If $B \cap N(x) \neq \emptyset$, say $w \in N(x) \cap B$, then $(A \cup\{w\}, B \backslash\{w\})$ is a monopolar partition of $G$. Hence, if $x \in B$, then $G$ has a monopolar partition different from $(A, B)$, which is a contradiction. So, $x \in A$.

Corollary 3.8 Let $G$ be a uniquely monopolar-partitionable block graph. Then no suspending block of $G$ is adjacent to a terminal block, an end star, or a terminal bowtie.

Proposition 3.9 Let $G^{\prime}$ be an induced subgraph of block graph $G$. Suppose that each monopolar partition of $G^{\prime}$ can be extended to at least one monopolar partition of $G$. Moreover, suppose that if $G^{\prime}$ has a unique monopolar partition, then it can be extended to exactly one monopolar partition of $G$. Then $G$ is uniquely monopolar-partitionable if and only if $G^{\prime}$ is.

Proof: Suppose that $G$ is uniquely monopolar-partitionable. Since $G^{\prime}$ is an induced subgraph of $G$, it follows that $G^{\prime}$ is a monopolar graph. If $G^{\prime}$ has two different monopolar partitions, then these monopolar partitions can be extended to two different monopolar partitions of $G$, which is a contradiction. Hence, $G^{\prime}$ is uniquely monopolar-partitionable.

Suppose that $G^{\prime}$ is uniquely monopolar-partitionable. It is obvious that $G$ is uniquely monopolarpartitionable.

By Proposition 3.7 and Proposition 3.9 we have the following corollary.

Corollary 3.10 Let $G$ be a block graph and let $G^{\prime}$ be defined as follows: 
- if $G$ contains a leaf $w$ adjacent to a block, then $G^{\prime}=G-w$;

- if a vertex $v$ is adjacent to two terminal blocks $Q_{1}$ and $Q_{2}$, then $G^{\prime}=G-Q_{2}$;

- if $G$ contains the tangent subgraph $R_{6}=R_{u}^{1}$, then $G^{\prime}$ is obtained from $G$ by deleting $V\left(R_{6}\right) \backslash\{u\}$.

Then $G$ is uniquely monopolar-partitionable if and only if $G^{\prime}$ is.

\section{Reductions of block graphs}

Let $G$ be a block graph. In view of Propositions 3.4 3.5 and 3.6 we may assume that $G$ satisfies the following two conditions:

- Each bowtie of $G$ is a terminal bowtie and $G$ has no two adjacent terminal bowties.

- $G$ has no induced subgraph $R_{i}$ for $i=1,2,3,4,5$, where $u$ and $v$ in $R_{3}$ do not belong to the same big block of $G$.

Let $T$ be a tree obtained from $G$ by contracting each terminal bowtie, end star, and big block, respectively. Let $v_{0} v_{1} \cdots v_{d}$ be a longest path of $T$. Let $V_{i}=\left\{u \in V(T) \mid d\left(u, v_{0}\right)=i\right\}$ for $i=0,1,2, \cdots, d$. Note that $\left(V_{0}, V_{1}, \cdots, V_{d}\right)$ is a vertex partition of $T$. From the vertex partition of $T$, we obtain a vertex partition $\left(V_{G}^{0}, V_{G}^{1}, \cdots, V_{G}^{d}\right)$ of $G$ as follows: $u \in V_{i}$ if and only if $u \in V_{G}^{i}$ or all the vertices of the corresponding terminal bowtie, end star or big block belong to $V_{G}^{i}$.

For each big block $Q$ of $G$, if the block belongs to $V_{G}^{i}$ of $G$, then $Q$ is called an $i^{\text {th }}$ level big block. Suppose that $Q$ is the $i^{t h}$ level big block. If $v \in V(Q)$ is adjacent to a vertex in $V_{G}^{i-1}$, then $v$ is called the upper vertex of $Q$, the other vertices are called down vertices of $Q$. For any vertex $v \in V_{G}^{i}$, if there exists a vertex $u \in V_{G}^{i-1}$ such that $v u \in E(G)$, then $u$ is called the parent of $v$. Both $Q$ and $v$ are called children of $u$.

In the section, a family of some special graphs $\left\{H_{i}, F_{j}, Y_{k} \mid 1 \leq i \leq 4,1 \leq j \leq 5, k=2,5\right\}$ is given in Fig. 2, Fig. 3 and Fig. 4. Say $d \geq 4$. The basic idea in the section is as follows: By employing a tree trimming techniques, we delete all the vertices of $V_{G}^{d}$. Firstly, we consider each component $C$ in the induced subgraph $G\left[V_{G}^{d-1} \cup V_{G}^{d}\right]$ having nonempty intersection with $V_{G}^{d}$. By local structure of $C$, if $C$ is not isomorphic to $H_{i}$ for $i \in\{1,2,3,4\}$, either we can determine that $G$ is not uniquely monopolarpartitionable or some blocks of $C$ are deleted. Secondly, we consider each component $C^{\prime}$ in the induced subgraph $G\left[V_{G}^{d-2} \cup V_{G}^{d-1} \cup V_{G}^{d}\right]$ containing $H_{i}$ as a tangent subgraph, where $i \in\{1,2,3,4\}$. By local structure of $C^{\prime}$, if $C^{\prime}$ is not isomorphic to $F_{i}$ for $i \in\{1,2,3,4,5\}$, either we can determine that $G$ is not uniquely monopolar-partitionable or some blocks of $C^{\prime}$ are deleted. Thirdly, we consider each component $C^{\prime \prime}$ in the induced subgraph $G\left[V_{G}^{d-3} \cup V_{G}^{d-2} \cup V_{G}^{d-1} \cup V_{G}^{d}\right]$ containing $F_{j}$ as a tangent subgraph, where $j \in\{1,2,3,4,5\}$. By local structure of $C^{\prime \prime}$, if $C^{\prime \prime}$ is not isomorphic to $Y_{k}$ for $k \in\{2,5\}$, either we can determine that $G$ is not uniquely monopolar-partitionable or some blocks of $C^{\prime \prime}$ are deleted. Finally, we consider each component $C^{\prime \prime \prime}$ in the induced subgraph $G\left[V_{G}^{d-4} \cup V_{G}^{d-3} \cup V_{G}^{d-2} \cup V_{G}^{d-1} \cup V_{G}^{d}\right]$ containing $Y_{j}$ as a tangent subgraph, where $j \in\{2,5\}$. By local structure of $C^{\prime \prime \prime}$, either we can determine that $G$ is not uniquely monopolar-partitionable or all blocks of $C^{\prime \prime \prime}$ belonging to $V_{G}^{d}$ are deleted. Then we obtain a new block graph whose associated tree has diameter less than $d$.

Proposition 4.1 Let $Q$ be a big block of $G$, and let $G_{1}, G_{2}, \cdots, G_{t}$ be the components of $G-Q$. Assume that the upper vertex $v$ of $Q$ is adjacent to $G_{1}, \cdots, G_{s}$. Suppose that $G_{j}$ is a terminal bowtie, a terminal block, an end star or an isolated vertex for $j=s+1, \cdots, t$. 
(1) Suppose that there exists a down vertex $w$ of $Q$ such that $w$ is not adjacent to a terminal bowtie. Let $G^{\prime}=G\left[V(Q) \cup V\left(\bigcup_{1 \leq i \leq s} G_{i}\right)\right]$. Then $G$ is uniquely monopolar-partitionable if and only if $G^{\prime}$ is.

(2) Suppose that each down vertex of $Q$ is adjacent to a terminal bowtie. Let $G^{\prime}$ be obtained from $G$ by deleting all the $G_{k}$ except exactly one terminal bowtie for each down vertex, where $k \in$ $\{s+1, \cdots, t\}$. Then $G$ is uniquely monopolar-partitionable if and only if $G^{\prime}$ is.

Proof: (1) Suppose that $G$ is uniquely monopolar-partitionable. Since $G^{\prime}$ is an induced subgraph of $G, G^{\prime}$ is a monopolar graph. For any monopolar partition $\left(A^{\prime}, B^{\prime}\right)$ of $G^{\prime}, v \in A^{\prime}$. Otherwise, $G^{\prime}$ has at least two monopolar partitions. One has $V(Q) \subseteq B^{\prime}$, the other has $V(Q) \backslash\{w\} \subseteq B^{\prime}$ and $w \in A^{\prime}$. Both of them can be extended to a monopolar partition of $G$, which is a contradiction. Since $v \in A^{\prime}$, any monopolar partition $\left(A^{\prime}, B^{\prime}\right)$ of $G\left[V(Q) \cup V\left(\bigcup_{1 \leq i \leq s} G_{i}\right)\right]$ can be extended to exactly one monopolar partition of $G$. So $G^{\prime}$ is uniquely monopolar-partitionable.

Suppose that $G^{\prime}$ is uniquely monopolar-partitionable. Let $\left(A^{\prime}, B^{\prime}\right)$ be its monopolar partition. By Proposition 3.7 $v \in A^{\prime}$. Since $v \in A^{\prime},\left(A^{\prime}, B^{\prime}\right)$ can be extended to exactly one monopolar partition of $G$. By Proposition 3.9, $G$ is uniquely monopolar-partitionable.

(2) By Proposition 3.9, $G$ is uniquely monopolar-partitionable if and only if $G^{\prime}$ is.

Proposition 4.2 Let $v$ be a vertex of $G$ not belonging to any big block, and let $t$ denote the parent of $v$. Suppose $v$ is not adjacent to a terminal bowtie and every child of $v$ is a leaf, a terminal block or end star.

(1) If $G$ is uniquely monopolar-partitionable, then $v$ is not adjacent to an end star.

(2) If $G$ is uniquely monopolar-partitionable, then $v$ is not adjacent to both a leaf and a terminal block.

Proof: (1) Suppose $v$ is adjacent to an end star. Let $u$ and $w$ be the center and a leaf of the end star, respectively. Let $(A, B)$ be the unique monopolar partition of $G$. By Proposition 3.7, $u \in A$ and $v \in B$. If $t \in A$, then let $u \in B$ and $N(u) \cup N(v) \backslash\{u, v\} \subseteq A$. So there exists a monopolar partition such that $u \in B$, which is a contradiction. If $t \in B$, let $N(v) \subseteq B$ and $N(u) \subseteq A$, then there exists a monopolar partition such that $u \in B$, which is a contradiction.

(2) Suppose $v$ is adjacent to both a leaf $s$ and a terminal block. Let $u$ and $w$ be the upper vertex and a down vertex of the terminal block, respectively. Let $(A, B)$ be the unique monopolar partition of $G$. By Proposition 3.7 $u \in A$ and $v \in B$. If $t \in A$, then $s \in A$ or $s \in B$. Then $G$ has two different monopolar partitions, which is a contradiction. If $t \in B$, let $v \in A$ and $N[u] \backslash\{v\} \subseteq B$, then there exists a monopolar partition such that $u \in B$, which is a contradiction.

By Proposition 4.1, Proposition 4.2, Corollary 3.8, Corollary 3.10 and the fact that $G$ has no induced subgraph $R_{1}$ and $R_{5}$, if $d \geq 2$, then we can assume that each component of $G\left[V_{G}^{d-1} \cup V_{G}^{d}\right]$, having nonempty intersection with $V_{G}^{d}$, is isomorphic to $H_{i}$ for $i \in\{1,2,3,4\}$. 


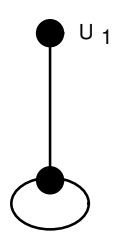

$\mathrm{H}_{1}$

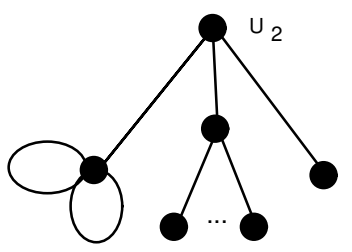

$\mathrm{H}_{2}$

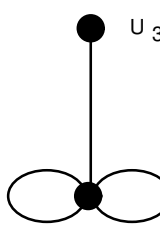

$\mathrm{H}_{3}$

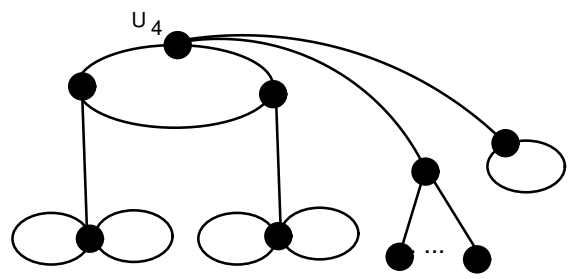

$\mathrm{H}_{4}$

Fig. 2. Each ellipse is a big block, $u_{2}$ is adjacent to at least one leaf or end star,

$u_{4}$ is adjacent to at least one end star or terminal block.

For each $H_{i}$, let $w_{i}$ denote the parent of $u_{i}$. For $i=1, \cdots, 4$, let $F_{i}=G\left[V\left(H_{i}\right) \cup\left\{w_{i}\right\}\right]$. If $w_{i}=w_{j}$, then let $F_{i j}=G\left[V\left(F_{i}\right) \cup V\left(F_{j}\right)\right]$. That is $F_{i j}=G\left[V\left(H_{i}\right) \cup V\left(H_{j}\right) \cup\left\{w_{i}\right\}\right]$. For $k=1,2,3,4$, let $F_{j}^{k}$ denote the graph obtained from $F_{j}$ by joining $w_{j}$ to a terminal bowtie, an end star, a terminal block and a leaf, respectively.

By a proof similar to that of Proposition 3.7, we have the following.

Proposition 4.3 Suppose that $(A, B)$ is the unique monopolar partition of block graph $G$. If $G$ contains the tangent subgraph $F_{i}=R_{w_{i}}^{1}$ for $i=1,4$, then $w_{i} \in A$. If $G$ contains the tangent subgraph $F_{2}=R_{w_{2}}^{1}$, then $w_{2} \in B$.

By Proposition 4.3 and Proposition 3.7, we have the following corollary.

Corollary 4.4 Let $G$ be uniquely monopolar-partitionable. Then $G$ does not contain tangent subgraph $F_{12}, F_{24}, F_{1}^{1}, F_{1}^{2}, F_{1}^{3}, F_{4}^{1}, F_{4}^{2}$ and $F_{4}^{3}$.

Proposition 4.5 If $G$ contains the tangent subgraph $F_{i j}$ for $i, j \in\{1,4\}$, then $G$ is uniquely monopolarpartitionable if and only if $G-H_{j}$ is.

Proof: It is obvious that $G-H_{j}$ is an induced subgraph of $G$. For any monopolar partition of $G-H_{j}$, it can be extended to at least a monopolar partition of $G$. Suppose that $G-H_{j}$ is uniquely monopolarpartitionable. Let $\left(A^{\prime}, B^{\prime}\right)$ be the unique monopolar partition of $G-H_{j}$. By Proposition $4.3, w_{i} \in A^{\prime}$. Then $\left(A^{\prime}, B^{\prime}\right)$ can be extended to exactly one monopolar partition of $G$. By Proposition 3.9, $G$ is uniquely monopolar-partitionable if and only if $G-H_{j}$ is.

By a proof similar to that of Proposition 4.5, we have the following.

Proposition 4.6 (1) For any $i \in\{1,2,3,4\}$, if $G$ contains the tangent subgraph $F_{i}^{4}$, then $G$ is uniquely monopolar-partitionable if and only if $G-t$ is, where $t \in V\left(F_{i}^{4}\right)$ is the leaf and is adjacent to $w_{i}$.

(2) Suppose that $G$ contains the tangent subgraph $F_{2}^{i}$ for $i=2,3$. Let $G^{\prime}$ be the graph obtained from $G$ by deleting the end star and the terminal block that are adjacent to $w_{2}$. Then $G$ is uniquely monopolarpartitionable if and only if $G^{\prime}$ is. 
Proposition 4.7 Suppose that $G$ contains the tangent subgraph $F_{3 j}$ for $j \in\{1,4\}$. Let $G^{\prime}$ be obtained from $G$ by deleting $F_{3 j} \backslash\left\{w_{3}\right\}$ and adhering a big block to $w_{3}$. Then $G$ is uniquely monopolarpartitionable if and only if $G^{\prime}$ is.

Proof: Let $G^{\prime \prime}=G-F_{3 j} \backslash\left\{w_{3}\right\}$. Suppose that $G$ is uniquely monopolar-partitionable. Let $(A, B)$ be the unique monopolar partition of $G$. By Proposition $4.3, w_{3} \in A$. Then $\left(A \cap V\left(G^{\prime \prime}\right), B \cap V\left(G^{\prime \prime}\right)\right)$ can be extended to a monopolar partition of $G^{\prime}$. Hence, $G^{\prime}$ is a monopolar graph. For any monopolar partition $\left(A^{\prime}, B^{\prime}\right)$ of $G^{\prime}, w_{3} \in A^{\prime}$. Otherwise, $\left(A^{\prime} \cap V\left(G^{\prime \prime}\right), B^{\prime} \cap V\left(G^{\prime \prime}\right)\right)$ can be extended to two different monopolar partitions of $G$, which is a contradiction. Since $w_{3} \in A^{\prime},\left(A^{\prime} \cap V\left(G^{\prime \prime}\right), B^{\prime} \cap V\left(G^{\prime \prime}\right)\right)$ can be extended to exactly one monopolar partition of $G$. Hence, $G^{\prime}$ is uniquely monopolar-partitionable.

Suppose that $G^{\prime}$ is uniquely monopolar-partitionable. Let $\left(A^{\prime}, B^{\prime}\right)$ be the unique monopolar partition of $G^{\prime}$. By Proposition 3.7 $w_{3} \in A^{\prime}$. Then $\left(A^{\prime} \cap V\left(G^{\prime \prime}\right), B^{\prime} \cap V\left(G^{\prime \prime}\right)\right)$ can be extended to a monopolar partition of $G$. Hence, $G$ is a monopolar graph. For any monopolar partition $(A, B)$ of $G, w_{3} \in A$. Otherwise, $G^{\prime}$ has two different monopolar partitions, which is a contradiction. If $G$ has two different monopolar partitions, then $G^{\prime \prime}$ has two different monopolar partitions. So $G^{\prime}$ has two different monopolar partitions, which is a contradiction. Hence, $G$ is uniquely monopolar-partitionable.

By Corollary 4.4, Proposition 4.5. Proposition 4.6. Proposition 4.7 and the fact that $G$ has no induced subgraph $R_{3}$ and $R_{4}$, we can assume that the subgraph induced by $w_{i}$ and its descendant, having nonempty intersection with $V_{G}^{d}$, is isomorphic to $F_{i}$ in Fig 3 for $i \in\{1, \cdots, 5\}$.

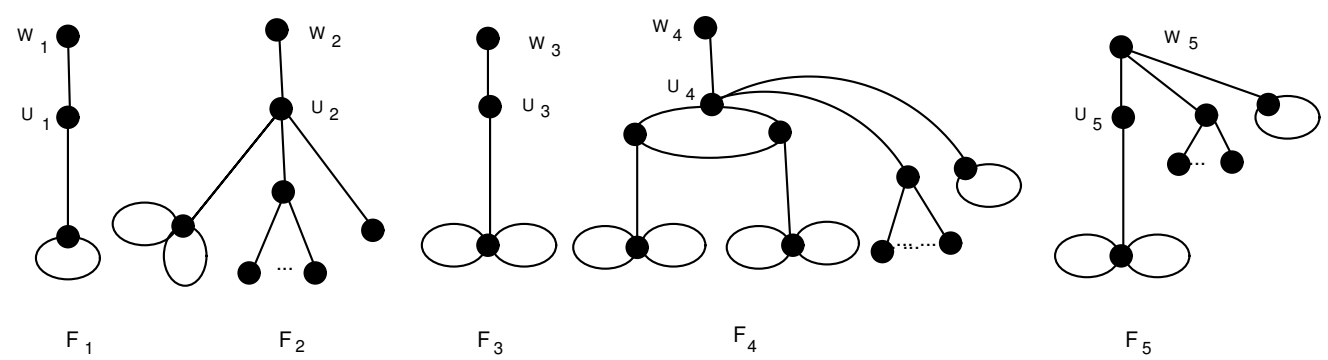

Fig. 3. $w_{5}$ is adjacent to at least one terminal block or an end star

Since $G$ has no induced subgraph $R_{1}, w_{2}, w_{3}, w_{5}$ do not belong to a big block. Let $R_{7}^{H_{i}}$ and $R_{8}^{H_{i}}$ be defined in Fig. 1 for $i \in\{1,4\}$. For each $F_{i}, i=1,4$, if $w_{i}$ belongs to a big block of $G$, then we have the following:

Proposition 4.8 Let $G$ be a block graph.

(1) Suppose that $G$ contains the tangent subgraph $F_{i}=R_{w_{i}}^{1}$, where $i \in\{1,4\}$. If $G$ is uniquely monopolar-partitionable, then $w_{i}$ is not a down vertex of a big block .

(2)Suppose that $G$ contains the tangent subgraph $R_{7}^{H_{i}}=R_{u}^{1}$, where $G\left[V\left(H_{i}\right) \cup\{v\}\right]=F_{i}$ and $i \in\{1,4\}$. Then $G$ is uniquely monopolar-partitionable if and only if $G-H_{i}$ is.

(3)Suppose that $G$ contains the tangent subgraph $R_{8}^{H_{i}}=R_{u}^{1}$, where $G\left[V\left(H_{i}\right) \cup\{v\}\right]=F_{i}$ and $i \in\{1,4\}$. Let $G^{\prime}$ be obtained from $G$ by deleting $H_{i}$ and all the children of the big block $Q$. Then $G$ is uniquely monopolar-partitionable if and only if $G^{\prime}$ is. 
Proof: (1) Suppose that $w_{i}$ is a down vertex of a big block. Let $(A, B)$ be the unique monopolar partition of $G$. By Proposition 4.3, $w_{i} \in A$. Let $G^{\prime}=G-H_{i}$. Then $\left(A \cap V\left(G^{\prime}\right), B \cap V\left(G^{\prime}\right)\right)$ is a monopolar partition of $G^{\prime}$. So $\left(A \cap\left(V\left(G^{\prime}\right)-w_{i}\right),\left(B \cap V\left(G^{\prime}\right)\right) \cup\left\{w_{i}\right\}\right)$ is also a monopolar partition of $G^{\prime}$ and it can be extended to a monopolar partition of $G$. Hence, $G$ has two different monopolar partitions, which is a contradiction.

(2) It is obvious that $G-H_{i}$ is an induced subgraph of $G$. For any monopolar partition $\left(A^{\prime}, B^{\prime}\right)$ of $G-H_{i}$, it can be extended to at least a monopolar partitions of $G$. Suppose that $G-H_{i}$ is uniquely monopolar-partitionable. Let $\left(A^{\prime}, B^{\prime}\right)$ be the unique monopolar partition. By Proposition 3.7 $v \in A^{\prime}$. Then $\left(A^{\prime}, B^{\prime}\right)$ can extend to exactly one monopolar partition of $G$. By Proposition 3.9 , $G$ is uniquely monopolar-partitionable if and only if $G-H_{i}$ is.

(3) A proof similar to that of Case 1 in Proposition 4.1 shows that $G$ is uniquely monopolar-partitionable if and only if $G^{\prime}$ is.

Let $m_{i}$ denote the parent of $w_{i}$ and $Y_{i}=G\left[V\left(F_{i}\right) \cup\left\{m_{i}\right\}\right]$ for $i=1,2, \cdots, 5$. If $m_{i}=w_{j}$, let $Y F_{i j}=G\left[V\left(Y_{i}\right) \cup V\left(F_{j}\right)\right]$ for $i=2,5$ and $j=1,2, \cdots, 5$. If $m_{i}=m_{j}$, let $Y_{i j}=G\left[V\left(Y_{i}\right) \cup V\left(Y_{j}\right)\right]$ for $i, j=2,5$. Let $Y_{i}^{j}$ be the graph obtained from $Y_{i}$ by joining $m_{i}$ to a terminal bowtie, an end star, a terminal block or an isolated vertex, respectively, for $i=2,5$ and $j=1,2,3,4$. By Proposition 4.8, we can assume that $Y_{i}=R_{m_{i}}^{1}$ is a tangent subgraph of $G$ for $i \in\{1, \cdots, 5\}$.

Proposition 4.9 Suppose that $G$ contains the tangent subgraph $Y_{i}$, where $i \in\{1,4\}$. Let $H_{i}$ be the tangent subgraph of $Y_{i}$. Then $G$ is uniquely monopolar-partitionable if and only if $G-H_{i} \backslash\left\{u_{i}\right\}$ is.

Proof: It is obvious that $G-H_{i} \backslash\left\{u_{i}\right\}$ is an induced subgraph of $G$. For any monopolar partition of $G-H_{i} \backslash\left\{u_{i}\right\}$ can be extended to at least a monopolar partition of $G$. Suppose that $G-H_{i} \backslash\left\{u_{i}\right\}$ is uniquely monopolar-partitionable. Let $\left(A^{\prime}, B^{\prime}\right)$ be the unique monopolar partition. By Proposition 3.7 . $w_{i} \in A^{\prime}$ and $u_{i} \in B^{\prime}$. Then $\left(A^{\prime}, B^{\prime}\right)$ can extend to exactly one monopolar partition of $G$. By Proposition 3.9. $G$ is uniquely monopolar-partitionable if and only if $G-H_{i} \backslash\left\{u_{i}\right\}$ is.

Proposition 4.10 Suppose that $G$ contains the tangent subgraph $Y_{3}$. Let $F_{3}$ be the tangent subgraph of $Y_{3}$. Then $G$ is uniquely monopolar-partitionable if and only if $G-F_{3}$ is.

Proof: By Proposition 3.9, $G$ is uniquely monopolar-partitionable if and only if $G-F_{3}$ is.
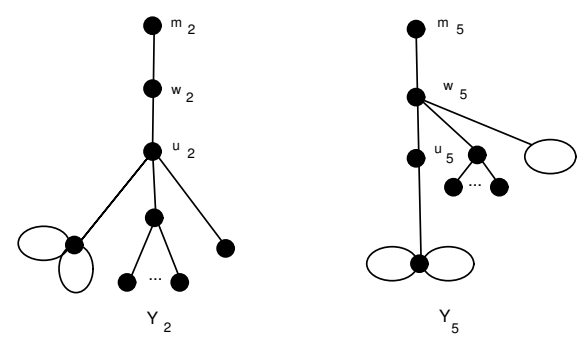

Fig. 4. Subgraphs $Y_{2}$ and $Y_{5}$ 
By Proposition 3.7 and Proposition 4.3, we have the following corollary.

Corollary 4.11 Suppose that block graph $G$ has unique monopolar partition $(A, B)$. If $G$ contains the tangent subgraph $Y_{i}$ for $i=2,5$, then $m_{i} \in A$.

By Propositions 4.3 and Corollary 4.11, we have the following corollary.

Corollary 4.12 Let $G$ be uniquely monopolar-partitionable block graph. Then $G$ does not contain tangent subgraph $Y F_{22}, Y F_{25}, Y F_{52}, Y F_{55}, Y_{2}^{j}$ and $Y_{5}^{j}$ for $j=1,2,3$.

By a proof similar to that of Proposition 4.5, we have the following.

Proposition 4.13 (1) For $i=2,5$ and $j=1,4$, suppose that $G$ contains the tangent subgraph $Y F_{i j}$. Let $H_{j}$ be the tangent subgraph of $F_{j}$. Then $G$ is uniquely monopolar-partitionable if and only if $G-H_{j}$ is.

(2) For $i, j=2,5$, suppose that $G$ contains the tangent subgraph $Y_{i j}$. Let $F_{j}$ be the tangent subgraph of $Y_{i}$. Then $G$ is uniquely monopolar-partitionable if and only if $G-F_{j}$ is.

(3) For $i=2,5$, if $G$ contains the tangent subgraph $Y_{i}^{4}$, then $G$ is uniquely monopolar-partitionable if and only if $G-t$ is, where $t \in V\left(Y_{i}^{4}\right)$ is a leaf and is adjacent to $m_{i}$.

By a proof similar to that of Proposition 4.7, we have the following.

Proposition 4.14 Suppose that $G$ contains the tangent subgraph $Y F_{i 3}$ for $i \in\{2,5\}$. Let $G^{\prime}$ be obtained from $G$ by deleting $Y F_{i 3} \backslash\left\{w_{3}\right\}$ and adhering a big block to $w_{3}$. Then $G$ is uniquely monopolarpartitionable if and only if $G^{\prime}$ is.

Let $R_{7}^{F_{i}}$ and $R_{8}^{F_{i}}$ be defined in Fig. 1 for $i \in\{2,5\}$. By a proof similar to that of Proposition 4.8, we have the following.

Proposition 4.15 Let $G$ be a block graph.

(1) Suppose that $G$ contains the tangent subgraph $Y_{i}$. If $G$ is uniquely monopolar-partitionable block graph, then $m_{i}$ is not a down vertex of a big block, where $i \in\{2,5\}$.

(2)Suppose that $G$ contains the tangent subgraph $R_{7}^{F_{i}}=R_{u}^{1}$, where $G\left[V\left(F_{i}\right) \cup\{v\}\right]=Y_{i}$ and $i \in$ $\{2,5\}$. Then $G$ is uniquely monopolar-partitionable if and only if $G-F_{i}$ is.

(3)Suppose that $G$ contains the tangent subgraph $R_{8}^{F_{i}}=R_{u}^{1}$, where $G\left[V\left(F_{i}\right) \cup\{v\}\right]=Y_{i}$ and $i \in$ $\{2,5\}$. Let $G^{\prime}$ be obtained from $G$ by deleting $F_{i}$ and all the children of the big block $Q$. Then $G$ is uniquely monopolar-partitionable if and only if $G^{\prime}$ is.

Let $t_{i}$ denote the parent of $m_{i}$ and $X_{i}=G\left[V\left(Y_{i}\right) \cup\left\{t_{i}\right\}\right]$ for $i=2,5$. By Corollary 4.12, Proposition 4.13. Proposition 4.14 and Proposition 4.15, we can assume that $X_{i}=R_{t_{i}}^{1}$ is a tangent subgraph of $G$ for $i \in\{2,5\}$.

By a proof similar to that of Proposition 4.1, we have the following.

Proposition 4.16 For $i=2,5$, suppose that $G$ contains the tangent subgraph $X_{i}$. Let $F_{i}$ be the tangent subgraph of $X_{i}$. Then $G$ is uniquely monopolar-partitionable if and only if $G-F_{i} \backslash\left\{w_{i}\right\}$ is.

Remark. By above Propositions, we have deleted all of vertices of $V_{G}^{d}$. So we obtain a new block graph whose associated tree has diameter less than $d$. 


\section{Uniquely monopolar-partitionable block graphs}

In order to determine whether a given block graph has a unique monopolar partition, we first define a family of block graphs. Let $\Phi$ be the family of block graphs $G$ satisfying the following conditions:

(1) Either $G$ is a bowtie or each bowtie of $G$ is a terminal bowtie.

(2) $G$ has no induced subgraph $R_{i}$ for $i=1,2,3,4,5$, where $u$ and $v$ in $R_{3}$ do not belong to the same big block of $G$.

(3) $G$ has no two adjacent terminal bowties.

Let $G$ be a block graph. By Proposition 3.4, if $G$ has two bowties $G\left[V\left(Q_{1} \cup Q_{2}\right)\right]$ and $G\left[V\left(Q_{2} \cup Q_{3}\right)\right]$ with different centers, then $G$ is not uniquely monopolar-partitionable. Obviously, if $G$ has two adjacent terminal bowties, then $G$ is not uniquely monopolar-partitionable. Without loss of generality, we can assume that $G$ has neither two bowties $G\left[V\left(Q_{1} \cup Q_{2}\right)\right]$ and $G\left[V\left(Q_{2} \cup Q_{3}\right)\right]$ with different centers nor two adjacent terminal bowties. By repeatedly applying Proposition 3.5 and Proposition 3.6, we obtain block graph $G_{1}, \cdots, G_{t}$ such that $G_{i} \in \Phi$ or $G_{i} \in\left\{\right.$ a flower, $\left.R_{3}, R_{5}\right\}$ for $1 \leq i \leq t$. We have the following.

Theorem 5.1 Let $G$ be a block graph. Suppose that $G$ has neither two bowties $G\left[V\left(Q_{1} \cup Q_{2}\right)\right]$ and $G\left[V\left(Q_{2} \cup Q_{3}\right)\right]$ with different centers nor two adjacent terminal bowties. Then $G$ is uniquely monopolarpartitionable if and only if $G_{i}$ is uniquely monopolar-partitionable for $1 \leq i \leq t$, where $G_{i}$ is defined as above.

If $G_{i}$ is a flower or $G_{i} \in\left\{R_{3}, R_{5}\right\}$, then $G_{i}$ is uniquely monopolar-partitionable. Now we determine whether or not a given block graph in $\Phi$ is uniquely monopolar-partitionable. Suppose that $G$ is a block graph and $G \in \Phi$. Let $T$ denote the tree structure of $G$. Now we define some operations on $G$ as follows:

Operation $\tau_{1}$ : If a block is adjacent to a leaf, delete the leaf; If a vertex $v$ is adjacent to two terminal blocks, delete one terminal block; If $G$ contains the tangent subgraph $R_{6}=R_{u}^{1}$, delete $V\left(R_{6}\right) \backslash\{u\}$.

Operation $\tau_{2}$ : Suppose that each down vertex of a big block is only adjacent to a leaf, a terminal bowtie, a terminal block or an end star. If there exists a down vertex such that it is not adjacent to a terminal bowtie, then delete all the children of each down vertex of the block; otherwise, delete all the children of each down vertex except one terminal bowtie.

Operation $\tau_{3}$ : Suppose that $i=1,4$. If $G$ contains the tangent subgraph $F_{i j}$ for $j=1$, delete $H_{j}$; If $G$ contains tangent subgraph $F_{j}^{4}$ for $j=1,2,3,4$, delete the leaf; If $G$ contains tangent subgraph $F_{3 i}$, delete $H_{i} \cup H_{3}$ and adhere a big block to $w_{3}$; If $G$ contains tangent subgraph $F_{2}^{j}$ for $j=2,3$, delete the end star and the terminal block; If $G$ contains the tangent subgraph $Y_{i}$, delete $V\left(H_{i}\right) \backslash\left\{u_{i}\right\}$; If $G$ contains the tangent subgraph $Y_{3}$, delete $F_{3}$.

Operation $\tau_{4}$ : Suppose that $i=2,5$. If $G$ contains tangent the subgraph $Y F_{i j}$ for $j=1,4$, delete $H_{j}$; If $G$ contains tangent subgraph $Y F_{3 i}$, delete $F_{i} \cup H_{3}$ and adhere a big block to $m_{3}$; If $G$ contains the tangent subgraph $Y_{i j}$ for $j=2,5$, delete $F_{j}$; If $G$ contains the tangent subgraph $Y_{i}^{4}$, delete the leaf; If $G$ contains the tangent subgraph $X_{i}$, delete $V\left(F_{i}\right) \backslash\left\{w_{i}\right\}$.

Operation $\tau_{5}$ : For $i \in\{1,4\}$, if $G$ contains the tangent subgraph $R_{7}^{H_{i}}=R_{u}^{1}$, delete $H_{i}$; if $G$ contains the tangent subgraph $R_{8}^{H_{i}}=R_{u}^{1}$, delete all the children of the big block of $R_{8}^{H_{i}}$. For $i \in\{2,5\}$, if $G$ contains the tangent subgraph $R_{7}^{F_{i}}=R_{u}^{1}$, delete $F_{i}$; if $G$ contains the tangent subgraph $R_{8}^{F_{i}}=R_{u}^{1}$, delete all the children of the big block of $R_{8}^{F_{i}}$.

By Propositions in Section 3 and Section 4, we have the following.

Theorem 5.2 Let $G^{\prime}$ be the graph obtained from $G \in \Phi$ by some operation $\tau_{i}$ for $i \in\{1, \cdots, 5\}$. Then $G$ is uniquely monopolar-partitionable if and only if $G^{\prime}$ is. 
Let $G^{*}$ be the graph obtained from $G \in \Phi$ by a series of operations $\tau_{i}$, where $i \in\{1, \cdots, 5\}$. It is obvious that $G^{*} \in \Phi$.

Theorem 5.3 Let $G \in \Phi$ with diam $(T) \leq 1$, where $T$ denotes the tree structure of $G$. Then $G$ is uniquely monopolar-partitionable if and only if $G$ is isomorphic to a bowtie or $\mathrm{H}_{3}$.

To present our algorithm for recognizing uniquely monopolar-partitionable block graphs, we introduce the following five properties $P_{i}$ and two sets $\mathcal{G}_{i}$ of graphs:

$P_{1}$ : there exists a vertex $v$ and a component $C$ of $G-v$ such that $G[V(C) \cup\{v\}]$ is a tree and is not a star;

$P_{2}$ : some suspending block is adjacent to a terminal block, an end star or a terminal bowtie;

$P_{3}$ : there exists a vertex $v$ such that $v$ neither belongs to a big block nor adjacent to a terminal bowtie, but $v$ is adjacent to either an end star or adjacent to both a leaf and a terminal block;

$P_{4}$ : for $i \in\{1,4\}, F_{i}$ is a tangent subgraph of $G$ and $w_{i}$ is a down vertex of a big block;

$P_{5}$ : for $j \in\{2,5\}, Y_{j}$ is a tangent subgraph of $G$ and $m_{j}$ is a down vertex of a big block.

$\mathcal{G}_{1}=\left\{F_{12}, F_{24}, Y F_{22}, Y F_{25}, Y F_{52}, Y F_{55}, F_{i}^{j}, Y_{k}^{j} \mid i=1,4 k=2,5, j=1,2,3\right\}$

$\mathcal{G}_{2}=\left\{F_{1}, F_{2}, F_{3}, H_{4}, Y_{2}, Y_{5}\right\}$

\section{Algorithm}

Input: A connected block graph $G \in \Phi$. Let $T$ denote the tree structure of $G$, and let $v_{0} v_{1} \cdots v_{d}$ be a longest path of $T$ and $\left(V_{G}^{0}, V_{G}^{1}, \cdots, V_{G}^{d}\right)$ be a vertex partition of $G$ according to $T$.

Output: Determine whether or not $G$ is uniquely monopolar-partitionable.

Repeatedly apply operation $\tau_{i}$ for $i=1, \cdots, 5$, until one of the following occurs

- $G$ has property $P_{i}$ for $i \in\{1, \cdots, 5\}$ (// $G$ is not uniquely monopolar-partitionable);

- $G$ contains a graph in $\mathcal{G}_{1}$ as a tangent subgraph (// $G$ is not uniquely monopolar-partitionable);

- the reduced graph is in $\mathcal{G}_{2}$ (// $G$ is not uniquely monopolar-partitionable);

- $\operatorname{diam}(T) \leq 1$ (// If $\operatorname{diam}(T) \leq 1$ and $G$ is isomorphic to a bowtie or to $H_{3}$, then $G$ is uniquely monopolar-partitionable; otherwise $G$ is not uniquely monopolar-partitionable).

We now discuss the correctness of the algorithm. In applying operations $\tau_{i}$, if any property $P_{i}$ occurs, then $G$ is not uniquely monopolar-partitionable according to Proposition 3.3 , Corollary 3.8 , and Propositions 4.2, 4.8 and 4.15 , if some graph in $\mathcal{G}_{1}$ is a tangent subgraph of $G$, then by Corollaries 4.4 and 4.12, $G$ is not uniquely monopolar-partitionable. Suppose that none of properties $P_{i}$ occurs and $G$ does not contain any graph of $\mathcal{G}_{1}$ as a tangent subgraph. Then the operations applied to $G$ yield either a graph in $\mathcal{G}_{2}$ or a graph whose associated $T$ has diameter at most one. If the reduced graph is in $\mathcal{G}_{2}$, then it is obvious that $G$ is not uniquely monopolar-partitionable. When $\operatorname{diam}(T) \leq 1$, by Theorem 5.3, $G$ is uniquely monopolar-partitionable if it is isomorphic to a bowtie or to $H_{3}$; otherwise $G$ is not uniquely monopolar-partitionable. Moreover all these steps can be implemented in polynomial time. Therefore we have the following:

Theorem 5.4 There is a polynomial time algorithm to decide if an input block graph is uniquely monopolar-partitionable. 


\section{References}

[1] H-J Bandelt and H.M. Mulder, Distance-hereditary graphs, J. Combinatorial Theory B 41 (1986) 182 - 208.

[2] Z.A. Chernyak and A.A. Chernyak, About recognizing $(\alpha, \beta)$-classes of polar graphs, Discrete Math. 62 (1986) $133-138$.

[3] R. Churchley and J. Huang, Line-polar graphs: characterization and recognition, SIAM Journal on Discrete Mathematics 25 (2011) 1269 - 1284.

[4] R. Churchley and J. Huang, List monopolar partitions of claw-free graphs, Discrete Mathematics (2011) 2545 - 2549.

[5] R. Churchley and J. Huang, Solving partition problems with colour-bipartitions, Graphs and Combinatorics (2012) DOI 10.1007/s00373-012-1270-z.

[6] R. Churchley and J. Huang, On the polarity and monopolarity of graphs, Journal of Graph Theory (2013) DOI 10.1002/jgt.21755.

[7] T. Ekim, P. Heggernes, and D. Meister, Polar permutation graphs, J. Fiala, J. Kratochvil, and M. Miller (eds.): IWOCA 2009, LNCS 5874, pp 218 - 229, 2009.

[8] T. Ekim, P. Hell, J. Stacho, and, D. de Werra, Polarity of chordal graphs, Discrete Applied Math. 156 (2008) $2469-2479$.

[9] T. Ekim and J. Huang, Recognizing line-polar bipartite graphs in time $O(n)$, Discrete Applied Math. 158 (2010) 1593 - 1598.

[10] T. Ekim, N.V.R. Mahadev and D. de Werra, Polar cographs, Discrete Applied Math. 156 (2008) 1652 - 1660.

[11] A. Farrugia, Vertex-partitioning into fixed additive induced-hereditary properties is NP-hard, Electronic J. Combinatorics 11 (2004).

[12] S. Foldes and P.L. Hammer, Split graphs, Proc. 8th South-Eastern Conf. on Combinatorics, Graph Theory and Computing (1977) 311 - 315.

[13] M.R. Garey and D.S. Johnson, Computers and Intractability, W.H. Freeman and Company, San Francisco (1979)., Graph Theory and Computing (1977) 311 - 315.

[14] M.C. Golumbic, Algorithmic Graph Theory and Perfect Graphs, Academic Press, New York, 1980.

[15] F. Harary, A characterization of block graphs, Canadian Mathematical Bulletin 6 (1963) 1 - 6.

[16] J. Huang and B. Xu, A forbidden subgraph characterization of line-polar bipartite graphs, Discrete Applied Math. 158 (2010) 666 - 680.

[17] R.I. Tyshkevich and A.A. Chernyak, Decompositions of graphs, Cybernetics and System Analysis 21 (1985) $231-242$.

[18] D. West, Introduction to Graph Theory, Second Edition, Prentice Hall, 2001. 